\title{
Estradiol Benzoate
}

National Cancer Institute

\section{Source}

National Cancer Institute. Estradiol Benzoate. NCI Thesaurus. Code C29769.

The synthetic benzoate ester of estradiol, a steroid sex hormone vital to the

maintenance of fertility and secondary sexual characteristics in females. As the primary,

most potent estrogen hormone produced by the ovaries, estradiol binds to and activates specific nuclear receptors. This agent exhibits mild anabolic and metabolic properties, and increases blood coagulability. ( $\mathrm{NCl04)}$ 